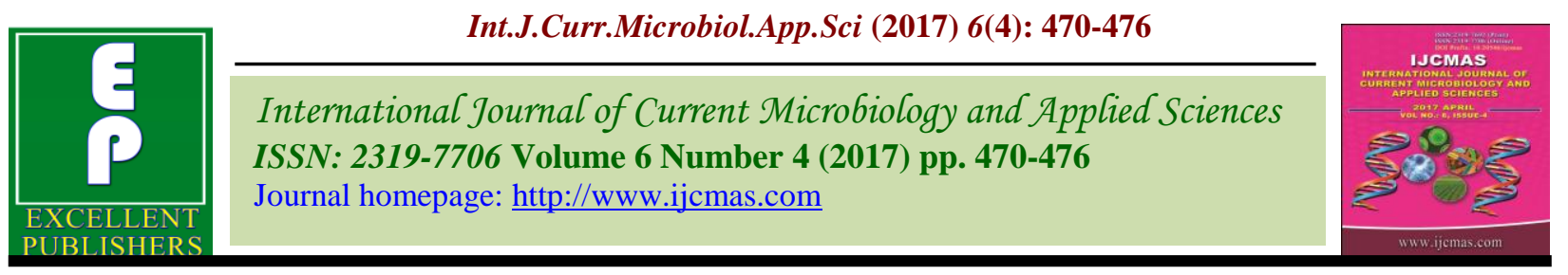

Original Research Article

https://doi.org/10.20546/ijcmas.2017.604.055

\title{
Combining Ability Analysis of Morpho-Physiological Traitsin Maize (Zea mays L.) inbred lines
}

\author{
Padma Lay* and A.K. Razdan \\ Division of Genetics and plant breeding, Sher-e-Kashmir University of Agricultural Sciences \\ and Technology (SKUAST-J), Chatha, Jammu \\ *Corresponding author
}

\section{A B S T R A C T}

Keywords

Maize (Zea mays L.), sca/gca, Gene action, Hybrids, Yield.

Article Info

Accepted:

02 March 2017

Available Online:

10 April 2017
Combining ability analysis for yield and its component traits was performed with 8lines, 3 testers and the resulting 24 crosses using LinexTester analysis. The analysis of variance indicated significant differences among parents, lines, testers, crosses, and parents vs. crosses for almost all the traits. The ratio of sca/gca variance revealed that there was preponderance of non additive gene action in the expression of all the traits. Inbred lines viz., JML-109, CML-161, JML 112 and V25 were good general combiners for yield and yield attributing characters, Tester VQL1 was high combiner for grain yield. Among the hybrids, CML-161 X CM 212, JML-109 X VQL1 and JM-112 X V341exhibited highest significant sca effects for yield and yield attributing traits.

\section{Introduction}

Maize is an extensively investigated crop for heterosis breeding and occupies the pride place in launching the first hybrid breeding programme in the country under auspicious of all India Co-ordinated Maize Improvement Project. Maize acreage and production have an increasing tendency with the introduction of hybrids due to its high yield potential. The nature and magnitude of gene action is an important factor in developing an effective breeding programme. Combining ability analysis is useful to assess the potential inbred lines and also helps in identifying the nature of gene action involved in various quantitative characters. This information is helpful to plant breeders for formulating hybrid breeding programmes. A wide array of biometrical tools is available to breeders for characterizing genetic control of economically important traits as a guide to decide upon an appropriate breeding methodology to involve in hybrid breeding. The present investigation was carried out to determine breeding value of genotypes, nature and magnitude of gene action for various yield and other important traits in maize (Zea mays L.). Linextester mating design developed by Kempthorne (1957), which provides reliable information on the general and specific combining ability effects of parents and their hybrid combinations was used to generate the information. The design has been widely used in maize by several workers and continues to be applied in 
quantitative genetic studies in maize (Joshi et al., 2002; Sharma et al., 2004).

\section{Materials and Methods}

Eight lines and three testers were mated in alinextester design during kharif2013. The resulting $24 \mathrm{~F} 1 \mathrm{~s}$ and their parents were grown in a Randomized Complete Block Design with two replications at farm of division of Plant Breeding and Genetics, SKUAST-J, chatha maintaining spacing between rows 60 $\mathrm{cm}$ and between plants $25 \mathrm{~cm}$ and one plant per hill. Observations were recorded on twelve yield and yield attributing traits viz., days to 50 per cent pollen shed, days to 50 per cent silking, days to 75 per cent brown husk plant height $(\mathrm{cm})$, ear placement height $(\mathrm{cm})$, Leaf width $(\mathrm{cm})$, ear length $(\mathrm{cm})$, ear girth $(\mathrm{cm})$, Kernel rows per year, Kernels per row Test weight $(\mathrm{g})$ and Grain yield plant ${ }^{-1}(\mathrm{~g})$. The data were subjected for analysis of variance for all the characters studied as per the method suggested by Panse and Sukhatme (1961). The variance of combining ability was estimated as per the procedure developed by Kempthorne (1957). The mean squares for GCA and SCA were tested against desired error variance.

\section{Results and Discussion}

Analysis of variance for line $\mathrm{x}$ tester revealed significant differences for line for all the characters, mean squares due to testers were significant in characters grain yield and ear girth. Variance components due to line $\mathrm{x}$ tester interaction showed significant in characters viz., ear placement height and leaf width (where variance due to lines was more). It has been generally reported that in relatively selected material non-additive gene action was more important than additive gene action (Sprague and Tatum, 1942 and Hayman, 1957). The experimental material generated from the crosses revealed the predominance of non-additive gene action for grain yield. Similar study confirms the findings of the workers (Divan et al., 2013; Kamara et al., 2014; Kanagrasu et al., 2010; Musa abadi et al., 2011). The analysis of variance for combining ability revealed that mean squares due to lines, testers and linextesters were significant for all the characters except days to $75 \%$ brown husk, ear placement height and test weight (Table 1). General combining ability effects for grain yield per plant, parents V358, JML-109, CML-161 and V341, JML-112 for Days to $50 \%$ pollen shed, CML-161 for days to 50\% silking, V358 for width, JML-109 and CML161 for ear length and ear girth JML-109, V25 and VQL1 for kernel per row were found to be good combiners this is in accordance with the studies of Divan et al., (2013) and Shah et al., (2015) days to 75\% brown husk, LTP-1.

Therefore, the parent V358 showed significant GCA effects for grain yield per plant and days to $75 \%$ brown husk; the parent JML-109 showed significant GCA effects for grain yield per plant, ear length and kernels per row; parent CML-161 showed significant GCA effects for grain yield per plant and ear girth; parent V358 showed significant GCA effects for grain yield per plant, days to $50 \%$ silking, days to $75 \%$ brown husk; parent $\mathrm{V}$ 341 showed significant GCA effects for Grain yield per plant and kernel rows per year (Table 2).

Among twelve characters studied all manifested higher degree of sca variance as compared to gca variance. The higher sca variance revealed the predominance of non additive genetic variance. Contrarily, importance of additive gene effects was reported by Alamnie et al., (2006). The per cent contribution of line $\mathrm{x}$ tester interaction appeared high to the bulk of the variation observed in hybrids. 
Table.1 Mean sum of squares in lines x testers design for 12 morpho-physiological traits in maize (Zea mays L.)

\begin{tabular}{|c|c|c|c|c|c|c|c|c|c|c|c|c|c|}
\hline $\begin{array}{l}\text { Source of } \\
\text { variation }\end{array}$ & d.f & $\begin{array}{l}\text { Grain } \\
\text { Yield } \\
(\text { gm) }\end{array}$ & $\begin{array}{c}\text { Days to } \\
50 \% \\
\text { Pollen } \\
\text { Shed }\end{array}$ & $\begin{array}{c}\text { Days to } \\
50 \% \\
\text { Silking }\end{array}$ & $\begin{array}{c}\text { Days to } \\
75 \% \\
\text { Brown } \\
\text { Husk }\end{array}$ & $\begin{array}{c}\text { Plant } \\
\text { Height }(\mathrm{cm})\end{array}$ & $\begin{array}{c}\text { Ear } \\
\text { Placement } \\
\text { Height } \\
(\mathbf{c m})\end{array}$ & $\begin{array}{c}\text { Leaf } \\
\text { Width } \\
(\mathrm{cm})\end{array}$ & $\begin{array}{c}\text { Ear } \\
\text { Length } \\
\text { (cm) }\end{array}$ & $\begin{array}{c}\text { Ear } \\
\text { Girth } \\
(\mathrm{cm})\end{array}$ & $\begin{array}{c}\text { Kernel } \\
\text { Rows } \\
\text { per } \\
\text { Ear }\end{array}$ & $\begin{array}{c}\text { Kernels } \\
\text { per } \\
\text { Row }\end{array}$ & $\begin{array}{c}\text { Test } \\
\text { Weight } \\
\text { (gm) }\end{array}$ \\
\hline Replication & 2 & 7.35 & 1.81 & 6.62 & 16.58 & 11.67 & 12.36 & 0.02 & 0.95 & 0.14 & 0.13 & 13.53 & 252.23 \\
\hline Treatment & 34 & $483.68 * *$ & $5.38 *$ & $4.67 *$ & $12.42 * *$ & $3549.83 * *$ & $930.50 * *$ & $0.46 * *$ & $5.97 * *$ & $1.04 * *$ & $1.34 * *$ & $41.68 * *$ & $455.16^{*}$ \\
\hline Parents & 10 & $231.69 * *$ & 5.03 & 5.11 & 8.84 & $132.79 *$ & 55.19 & $0.40 * *$ & $5.79 *$ & $1.23 *$ & 0.28 & 15.09 & 301.30 \\
\hline $\begin{array}{c}\text { Parents } \\
\text { (lines) }\end{array}$ & 7 & $159.63 * *$ & 5.10 & 5.33 & 7.52 & $173.39 *$ & 18.39 & $0.30 *$ & $6.06^{*}$ & 1.00 & 0.211 & 14.61 & 294.81 \\
\hline $\begin{array}{c}\text { Parents } \\
\text { (tester) }\end{array}$ & 2 & $589.82^{* *}$ & 4.44 & 5.10 & 9.16 & 55.98 & 8.191 & 0.15 & 7.62 & $2.24 *$ & 0.64 & 13.44 & 474.67 \\
\hline $\begin{array}{l}\text { Parents } \\
\text { (L vs T) }\end{array}$ & 1 & 19.89 & 5.77 & 3.67 & 17.37 & 2.14 & $406.78 * *$ & $1.61 * *$ & 0.22 & 0.84 & 0.02 & 21.70 & 0.065 \\
\hline $\begin{array}{c}\text { Parents vs. } \\
\text { crosses }\end{array}$ & 1 & $7421.16 * *$ & $81.97 * *$ & $42.96 * *$ & $175.97 * *$ & $110376.17 * *$ & $28027.30 * *$ & $7.25^{* *}$ & $79.47 * *$ & $7.36^{* *}$ & $16.82 * *$ & $496.01 * *$ & $5058.14 * *$ \\
\hline Crosses & 23 & $291.61 * *$ & 2.20 & 2.82 & 6.86 & $390.88 * *$ & $132.96 * *$ & 0.19 & 2.85 & 0.68 & $1.13 * *$ & $33.49 * *$ & 321.93 \\
\hline Error & 68 & 31.24 & 2.88 & 2.75 & 6.07 & 64.65 & 44.33 & 0.13 & 2.48 & 0.52 & 0.36 & 8.89 & 235.38 \\
\hline
\end{tabular}


Table.2 Estimates of General combining ability effects of lines and testers for different characters in maize (Zea mays L.)

\begin{tabular}{|c|c|c|c|c|c|c|c|c|c|c|c|c|}
\hline Source & $\begin{array}{l}\text { Grain } \\
\text { Yield } \\
(\text { gm) }\end{array}$ & $\begin{array}{c}\text { Days to } \\
50 \% \\
\text { Pollen } \\
\text { Shed }\end{array}$ & $\begin{array}{c}\text { Days to } \\
50 \% \\
\text { Silking }\end{array}$ & $\begin{array}{c}\text { Days to } \\
75 \% \\
\text { Brown } \\
\text { Husk }\end{array}$ & $\begin{array}{c}\text { Plant } \\
\text { Height } \\
\text { (cm) }\end{array}$ & $\begin{array}{c}\text { Ear } \\
\text { Placement } \\
\text { Height } \\
(\mathbf{c m})\end{array}$ & $\begin{array}{c}\text { Leaf } \\
\text { Width } \\
\text { (cm) }\end{array}$ & $\begin{array}{c}\text { Ear } \\
\text { Length } \\
\mathrm{cm}\end{array}$ & $\begin{array}{c}\text { Ear } \\
\text { Girth } \\
\text { cm }\end{array}$ & $\begin{array}{c}\text { Kernel } \\
\text { Rows } \\
\text { per Ear }\end{array}$ & $\begin{array}{c}\text { Kernels } \\
\text { per Row }\end{array}$ & $\begin{array}{c}\text { Test } \\
\text { Weight } \\
(\text { gm) }\end{array}$ \\
\hline \multicolumn{13}{|l|}{ Line } \\
\hline JML-109 & $7.49 * *$ & -0.35 & -0.04 & -0.58 & -3.11 & -1.17 & 0.02 & $1.41 *$ & 0.39 & 0.40 & $2.85 * *$ & 3.74 \\
\hline CML-161 & $5.38 * *$ & -0.42 & $-1.18^{*}$ & 0.29 & 5.59 & 0.21 & -0.06 & -0.02 & $0.64 *$ & 0.16 & $-3.27 * *$ & -2.08 \\
\hline V25 & $-10.54 * *$ & 0.53 & 0.90 & 0.51 & -0.57 & -1.33 & 0.18 & 0.72 & 0.26 & -0.12 & $2.63^{*}$ & -0.49 \\
\hline HKI-161 & -0.55 & 1.04 & 0.84 & 0.96 & 3.57 & 3.54 & -0.04 & -0.59 & -0.11 & -0.17 & 1.38 & 0.97 \\
\hline JML-120 & $-9.39 * *$ & -0.14 & 0.46 & -0.47 & -3.03 & -1.94 & -0.07 & -0.48 & -0.40 & $-0.43^{*}$ & -0.42 & -9.48 \\
\hline LTP-1 & $-4.85^{*}$ & 0.25 & -0.21 & 0.44 & $15.61 * *$ & $6.42 * *$ & 0.11 & -0.73 & -0.36 & 0.35 & -1.25 & 1.18 \\
\hline JML-112 & 0.01 & $-1.31 *$ & -0.20 & 0.73 & -4.19 & 1.01 & -0.14 & -0.67 & -0.44 & $-0.45 *$ & $-2.40 *$ & -0.36 \\
\hline V358 & $12.47 * *$ & 0.40 & -0.57 & $-1.87 *$ & $-13.86 * *$ & $-6.74 * *$ & 0.00 & 0.37 & 0.01 & 0.26 & 0.48 & 6.52 \\
\hline S.E.gi(line) & 1.89 & 0.57 & 0.56 & 0.82 & 2.68 & 2.22 & 0.12 & 0.52 & 0.24 & 0.20 & 0.99 & 5.11 \\
\hline S.E.gi-gj(line) & 2.67 & 0.80 & 0.79 & 1.16 & 3.79 & 3.13 & 0.17 & 0.74 & 0.34 & 0.29 & 1.40 & 7.23 \\
\hline \multicolumn{13}{|l|}{ Tester } \\
\hline V341 & $2.83^{*}$ & 0.02 & -0.50 & -0.09 & 1.97 & -0.30 & $-0.16^{*}$ & -0.11 & 0.05 & $0.31 *$ & -0.97 & 5.93 \\
\hline $\mathrm{CM} 212$ & -1.03 & -0.04 & 0.47 & 0.54 & 1.68 & 1.90 & 0.00 & 0.13 & 0.13 & 0.00 & -0.55 & -5.76 \\
\hline VQL1 & -1.80 & 0.02 & 0.04 & -0.45 & $-3.65^{*}$ & -1.60 & $0.16^{*}$ & -0.01 & -0.18 & $-0.30 *$ & $1.51 *$ & -0.17 \\
\hline S.E.gi(tester) & 1.15 & 0.35 & 0.34 & 0.50 & 1.64 & 1.36 & 0.08 & 0.32 & 0.15 & 0.12 & 0.61 & 3.13 \\
\hline S.E.gi-gj(tester) & 1.63 & 0.49 & 0.48 & 0.71 & 2.32 & 1.92 & 0.10 & 0.46 & 0.20 & 0.18 & 0.86 & 4.42 \\
\hline
\end{tabular}


Table.3 Estimates of Specific combining ability effects of crosses for different traits of $(3 \mathrm{x} 8)$ line $\mathrm{x}$ tester analysis

\begin{tabular}{|c|c|c|c|c|c|c|c|c|c|c|c|c|}
\hline Crosses & $\begin{array}{l}\text { Grain } \\
\text { Yield } \\
\text { (gm) }\end{array}$ & $\begin{array}{c}\text { Days to } \\
50 \% \\
\text { Pollen } \\
\text { Shed }\end{array}$ & $\begin{array}{c}\text { Days } \\
\text { to } \\
50 \% \\
\text { Silking }\end{array}$ & $\begin{array}{c}\text { Days } \\
\text { to } 75 \\
\% \\
\text { Brown } \\
\text { Husk } \\
\end{array}$ & $\begin{array}{c}\text { Plant } \\
\text { Height } \\
\text { (cm) }\end{array}$ & $\begin{array}{c}\text { Ear } \\
\text { placement } \\
\text { Height } \\
\text { (cm) }\end{array}$ & $\begin{array}{c}\text { Leaf } \\
\text { Width } \\
\text { (cm) }\end{array}$ & $\begin{array}{c}\text { Ear } \\
\text { Length } \\
\text { (cm) }\end{array}$ & $\begin{array}{c}\text { Ear } \\
\text { Girth } \\
\text { (cm) }\end{array}$ & $\begin{array}{c}\text { Kernel } \\
\text { Rows } \\
\text { per } \\
\text { Ear }\end{array}$ & $\begin{array}{c}\text { Kernels } \\
\text { per } \\
\text { Row }\end{array}$ & $\begin{array}{c}\text { Test } \\
\text { Weight } \\
\text { (gm) }\end{array}$ \\
\hline JML-109 xV341 & -0.98 & 0.52 & -0.21 & -0.32 & $-10.32 *$ & $-9.22 *$ & -0.25 & 0.18 & 0.20 & 0.48 & $-4.01 *$ & -2.99 \\
\hline JML-109 xCM212 & $-9.15 * *$ & -1.32 & -0.24 & 0.28 & $16.96 * *$ & $11.62 * *$ & -0.25 & -1.25 & -0.21 & $0.77 *$ & 1.91 & 1.72 \\
\hline JML-109 X VQL1 & $10.12 * *$ & 0.79 & 0.45 & 0.04 & -6.64 & -2.40 & $0.50 *$ & 1.06 & 0.01 & $-1.25 * *$ & 2.09 & 1.27 \\
\hline CML-161x V341 & $-10.03 * *$ & -0.23 & -0.31 & 2.84 & 1.05 & -0.81 & 0.16 & 0.24 & -0.08 & -0.52 & 1.96 & 3.83 \\
\hline CML-161x CM212 & $13.13 * *$ & -0.47 & -0.04 & 0.64 & -6.60 & -1.63 & 0.05 & -0.26 & -0.10 & -0.20 & 0.19 & -6.35 \\
\hline CML-161X VQL1 & -3.10 & 0.70 & 0.35 & $-3.47 *$ & 5.56 & 2.43 & -0.21 & 0.02 & 0.18 & $0.72 *$ & -2.15 & 2.52 \\
\hline $\mathrm{V} 25 \mathrm{xV} 341$ & -0.48 & 0.24 & -1.35 & -0.05 & $11.63 *$ & 5.33 & -0.14 & 0.12 & -0.53 & -0.33 & 2.55 & 13.44 \\
\hline V25xCM212 & -1.35 & 0.01 & 0.41 & -0.15 & -9.08 & -7.07 & -0.14 & -0.55 & 0.08 & -0.28 & 0.21 & -6.68 \\
\hline V25 x VQL1 & 1.82 & -0.25 & 0.94 & 0.20 & -2.55 & 1.74 & 0.28 & 0.43 & 0.45 & 0.61 & -2.76 & -6.76 \\
\hline HKI-161xV341 & 6.53 & 0.13 & 0.10 & -0.60 & 7.67 & 7.43 & -0.08 & -0.61 & -0.31 & -0.36 & -1.95 & -0.05 \\
\hline HKI-161xCM212 & -5.07 & 0.60 & -0.37 & -0.50 & -8.72 & $-7.98 *$ & 0.12 & 0.26 & 0.33 & 0.28 & 1.96 & -11.23 \\
\hline HKI-161x VQL1 & -1.47 & -0.73 & 0.26 & 1.09 & 1.05 & 0.55 & -0.03 & 0.35 & -0.02 & 0.08 & -0.01 & 11.28 \\
\hline JML-120 xV341 & -1.50 & -0.06 & 0.76 & -0.03 & 6.40 & 1.40 & -0.10 & -0.13 & 0.03 & -0.06 & -2.46 & 11.66 \\
\hline JML-120 xCM212 & 2.50 & 0.64 & -0.44 & 0.10 & 1.85 & 2.07 & 0.09 & 0.04 & -0.08 & 0.22 & -0.99 & -0.42 \\
\hline JML-120 xVQL1 & -1.00 & -0.58 & -0.32 & -0.07 & -8.25 & -3.47 & 0.00 & 0.10 & 0.05 & -0.16 & 3.45 & -11.24 \\
\hline LTP-1 xV341 & 0.77 & -0.21 & 0.80 & -1.54 & -1.44 & 0.69 & 0.08 & -0.42 & 0.09 & 0.26 & -0.13 & -8.83 \\
\hline LTP-1 xCM212 & -0.17 & 0.12 & -0.11 & -0.07 & -5.53 & -4.61 & -0.08 & 0.28 & -0.01 & -0.58 & $-3.48 *$ & 4.39 \\
\hline LTP-1 xVQL1 & -0.60 & 0.09 & -0.69 & 1.62 & 6.97 & 3.92 & 0.00 & 0.14 & -0.08 & 0.32 & $3.61 *$ & 4.44 \\
\hline JML-112xV341 & $9.91 * *$ & 0.09 & 0.62 & -0.60 & $-10.74^{*}$ & $-8.13 *$ & 0.14 & -0.12 & 0.16 & 0.25 & 0.49 & -5.89 \\
\hline JML-112xCM212 & -4.84 & -0.18 & 0.01 & 0.77 & 5.91 & $8.57 *$ & 0.11 & 1.39 & 0.27 & -0.11 & 0.83 & 1.99 \\
\hline JML-112 X VQL 1 & -5.06 & 0.09 & -0.63 & -0.17 & 4.84 & -0.44 & -0.24 & -1.26 & -0.43 & -0.14 & -1.32 & 3.90 \\
\hline V358xV341 & -4.22 & -0.49 & -0.42 & 0.30 & -4.24 & 3.32 & 0.20 & 0.74 & 0.45 & 0.29 & $3.53 *$ & -11.17 \\
\hline V358xCM212 & 4.94 & 0.61 & 0.78 & -1.06 & 5.21 & -0.98 & 0.10 & 0.11 & -0.28 & -0.11 & -0.64 & 16.58 \\
\hline V 358xVQL 1 & -0.72 & -0.12 & -0.36 & 0.76 & -0.96 & -2.33 & -0.30 & -0.84 & -0.16 & -0.18 & -2.90 & -5.41 \\
\hline S.E.(sij) & 3.26 & 0.98 & 0.96 & 1.42 & 4.64 & 3.84 & 0.20 & 0.91 & 0.42 & 0.35 & 1.72 & 8.86 \\
\hline S.E.sij-skl & 4.60 & 1.38 & 1.35 & 2.01 & 6.57 & 5.44 & 0.29 & 1.29 & 0.59 & 0.49 & 2.43 & 12.53 \\
\hline
\end{tabular}


High yield cross combination CML-161 X CM 212 showed significant SCA effects for grain yield per plant, days to $50 \%$ pollen shed and days to $50 \%$ silking; the cross JML-109 $X$ VQL1 showed significant SCA effects for grain yield per plant and leaf width; the cross JM -112 X V341 showed significant SCA effects for grain yield per plant. Good specific combiners (Table 3) were exhibited by crosses viz., CML-161 X CM 212, JML-109 $\mathrm{X}$ VQL1 and JML-112 X V341 for grain yield; CML-161 X VQL1 for days to $75 \%$ brown husk; JML-109 X CM 212 and V25 X V341 for plant height; JML-109 X CM 212 and JML-112 X CM 212 for ear placement height; LTP-1 X VQL1 and V358 X V341for Kernels per row. Therefore crosses viz., CML-161 X CM 212, JML-109 X VQL1 and JM-112 X V341 involved at least one parent as good general combiner indicated the superiority due to non additive gene action. These results are in agreement with Alamnie et al., (2003), Kamara et al., (2014), Mahesh et al., (2013) and Chandel and Mankotia (2014). Prasad and Pramod Kumar (2003), Subramaniyan and Subbraman (2006), Jayakumar and Sundram (2007), Vijayabharathi et al., (2009) reported that specific combining ability (SCA) variances were higher than general combining ability variances (GCA) for all the characters which indicated preponderance of non-additive gene action for all the characters.

\section{Acknowledgements}

The authors are thankful to the AICRP maize centre Udampur for supplying seed material to conduct this research.

\section{References}

Alamnie, A., Nayakar, N.Y. and Wali, M.C. 2003 Combining Ability, Heterosis and per se Performance of Height Characters in Maize. Karnataka J. Agri.
Sci., 16(1): 131-133.

Alamnie, A., Wali, M.C., Salimath, P.M. and Jagadeesha, R.C. 2006.Combining ability and heterosis for grain yield and ear characters in maize. Karnataka $J$. Agri. Sci., 19: 13-16.

Chandel, U. and Mankotia, B.S. 2014. Combining ability in local and CIMMYT inbred lines of maize (Zea mays L.) for grain yield and yield components using line $\times$ tester analysis. Sabrao J. Breeding and Genetics, 46(2): 256-264.

Divan, R., Khorasani, S.K., Ebrahimi, A. and Bakhtiari, S. 2013. Study on Combining Ability and Gene Effects in inbred lines and single Cross hybrids of Forage maize (Zea mays L.). Int. J. Agron. Plant Production, 4(6): 1290-1297.

Jayakumar, J. and Sundaram, T. 2007. Combining ability studies for grain yield and other yield components in maize (Zea mays L.), Crop Res., (Hisar), 33(1-3): 179-186.

Joshi, A.B. and Dhawan, N.L. 1966. Genetic improvement of yield with special reference to self-fertilizing crops. Indian J. Genetics, 26: 101-113.

Kamara, M.M., El-Degwy, I.S. and Koyama, H. 2014. Estimation combining ability of some maize inbred lines using line $x$ tester mating design under two nitrogen levels. Australian J. Crop Sci., 8(9): 1336-1342.

Kanagarasu, S., Nallathambi, G. and Ganesan, K.N. 2010. Combining ability analysis for yield and its component traits in maize (Zea mays L.). Electronic J. Plant Breeding, 1(4): 915-920.

Kempthorne, O. 1957. An Introduction to Genetics Statistics, 1st eds, pp. 457-71. John wiley and sons, New York.

Mahesh, N., Wali, M.C., Gowda, M.V.C., Motagi, B.N. and Uppinal, N.F. 2013. Genetic analysis of grain yield, starch, protein and oil content in single cross 
hybrids of maize. Karnataka J. Agri. Sci., 26(2): 185-189.

Musa-Abadi, J., Khavari-Khorasani, S., SyahSar, B., Movafeg, S. and Golbashi, M. 2011. Estimation of combining ability and gene effects in forage maize (Zea mays L.) using Line $\times$ Tester Crosses. $J$. Plant Physiol. Breeding, 1(1): 57-67.

Panse, V.G. and Sukhatme, P.V. 1961. Statistical methods for agricultural research workers, New Delhi: ICAR.

Prasad, S.K., and Kumar P., 2003, Linextester analysis for combining ability in maize, J. Res., (RAU), 13: 68-72.

Shah, L., Rahman, H.U., Ali, A., Bazai, N.A. and Tahir, M. 2015.Combining ability estimates from line $\mathrm{x}$ tester mating design in maize (Zea mays L.).
Academic Res. J. Agri. Sci. Res., 3(4): 71-75.

Subramaniyan, A., and Subbraman, N. 2006. Combining ability analysis for yield and its contributing traits in maize, Indian $J$. Agri. Res., 40: 131-134.

Vijayabharathi, A., Anandakumar, C.R., and Gnanamalar, R.P. 2009. Combining ability analysis for yield and its components in popcorn (Zea mays var. everta Sturt.). Electronic J. Plant Breeding, 1: 28-32.

Westman, A.L. and Kresovich, S. 1997. Use of molecular marker techniques for description of plant genetic variation. In: Callow, J. L., Ford-Lloyd, B. V. and Newburry, H. J. (eds.). Biotechnol. Plant Genetic Res., CAB Int., pp. 9-45

\section{How to cite this article:}

Padma Lay and Razdan, A.K. 2017. Combining Ability Analysis of Morpho-Physiological Traitsin Maize (Zea mays L.) inbred lines. Int.J.Curr.Microbiol.App.Sci. 6(4): 470-476. doi: https://doi.org/10.20546/ijcmas.2017.604.055 